\title{
Case Report \\ Perianal Basal Cell Carcinoma Successfully Managed with Excisional Biopsy
}

\author{
Paola C. Aldana $(\mathbb{D}){ }^{1}$ Harris G. Yfantis, ${ }^{1,2}$ and Preeti R. John $\mathbb{1 D}^{1,2}$ \\ ${ }^{1}$ University of Maryland School of Medicine, Baltimore, MD, USA \\ ${ }^{2}$ Baltimore Veterans Affairs Medical Center, Baltimore, MD, USA
}

Correspondence should be addressed to Paola C. Aldana; paola.aldana@som.umaryland.edu

Received 2 April 2019; Accepted 19 May 2019; Published 12 June 2019

Academic Editor: Ioannis D. Bassukas

Copyright (C) 2019 Paola C. Aldana et al. This is an open access article distributed under the Creative Commons Attribution License, which permits unrestricted use, distribution, and reproduction in any medium, provided the original work is properly cited.

\begin{abstract}
Basal cell carcinoma (BCC) is the most common cutaneous malignancy in the United States and is often nonaggressive. Its location in the perianal region is very rare and it is estimated that only $0.08 \%$ of all BCCs occur in this region. Herein, we present a case of perianal basal cell carcinoma, nodular type. The diagnosis was made using excisional biopsy of a skin lesion. Immunohistochemical staining confirmed the diagnosis: it showed diffuse and strong positivity for smooth muscle actin (SMA) and monoclonal antibody BER-Ep4 and was negative for carcinoembryonic antigen (CEA), pancytokeratin (AE1/AE3), and epithelial membrane antigen (EMA). The treatment of choice has traditionally been local excision to clear margins but the newest guidelines recommend Mohs Micrographic surgery (MMS) or standard 4mm surgical margins for this high-risk BCC. Our patient was successfully treated using excisional biopsy without recurrence. In select patients with lesions smaller than $1 \mathrm{~cm}$, excisional biopsy may be sufficient to treat the disease and may be better tolerated than MMS and wider surgical margins. Literature review suggests a predisposition for perianal BCC in individuals susceptible to cutaneous malignancies. Therefore, any history of cutaneous malignancy should further prompt clinicians to examine nonsun exposed areas on full body skin exams.
\end{abstract}

\section{Introduction}

Basal cell carcinoma (BCC) is the most common cutaneous malignancy in the United States and mostly frequently occurs on sun exposed areas such as the head and neck [1]. Although it can occur on other areas, involvement of the perianal skin and genitals is extremely rare [2]. It is estimated that perianal BCC accounts for only $0.08 \%$ of all BCC and $0.2 \%$ of all anorectal neoplasms $[1,3]$. In fact, there are less than 200 cases of BCC on the perianal skin and genitals reported in the literature [1]. We present a case of nodular perianal BCC that was diagnosed using immunohistochemical staining, for which excisional biopsy was performed with no subsequent recurrence.

\section{Case Presentation}

An 89-year-old male with no history of cutaneous malignancies was referred to General Surgery for evaluation of a persistent nodule on the skin of the right perianal region that he had noticed for more than a year. Past medical history was significant for gastric lymphoma (non-Hodgkins follicular type, with spontaneous remission).

He palpated the perianal lesion while cleaning the skin in this region. The lesion was not associated with bleeding, oozing, pain, or itching. He denied any concomitant skin lesion or rashes. He also denied fever, chills, or abdominal symptoms. On physical exam, a single small $0.5 \mathrm{~cm}$ cutaneous nodule with central dimpling and no discoloration was visualized in the perianal region. No concerning lesions were palpated on digital rectal exam. An excisional biopsy of the nodule was performed for histopathological examination.

Microscopic exam confirmed the diagnosis of perianal BCC, nodular type (Figure 1(a)). Histopathology revealed a lesion strongly and diffusely positive for immunohistochemical staining of smooth muscle actin (SMA) (Figure 1(b)) and negative for carcinoembryonic antigen (CEA) and pancytokeratin (AE1/AE3), supporting the diagnosis. Staining for epithelial membrane antigen (EMA) was negative in the tumor cells while monoclonal antibody BER-Ep4 displayed 


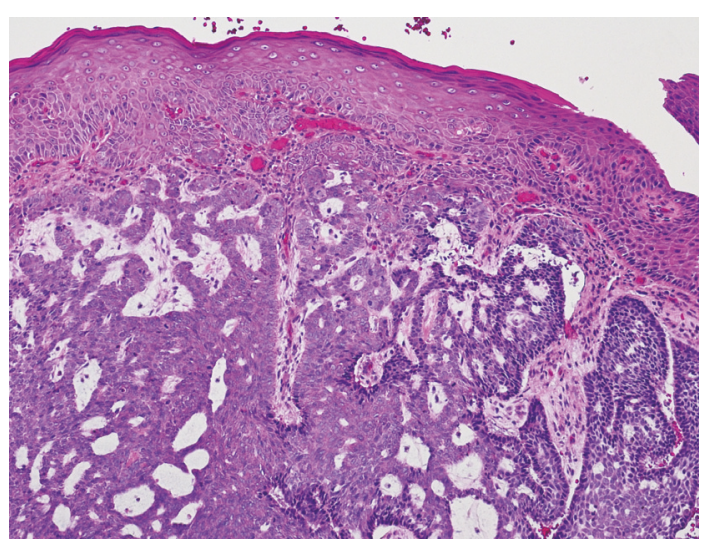

(a)

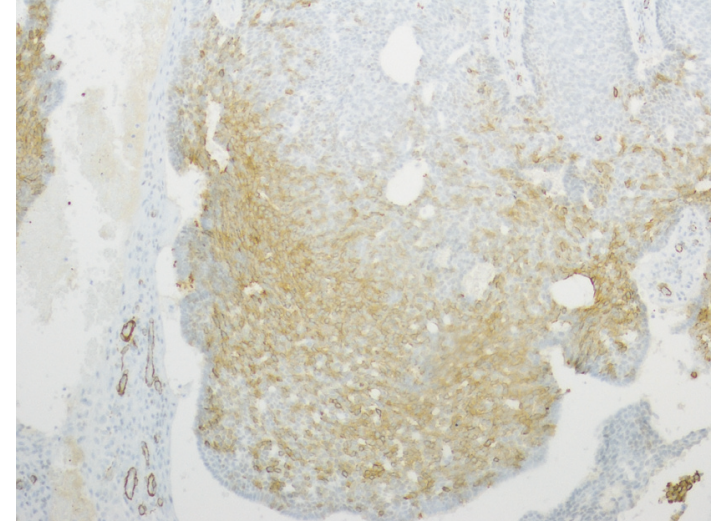

(b)

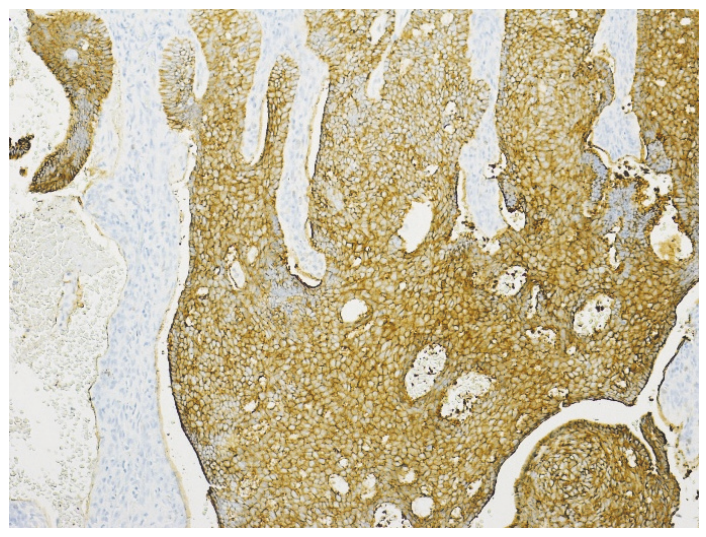

(c)

FIGURE 1: Histopathological analysis of perianal nodule biopsy. (a) Hematoxylin and Eosin (H\&E) stain (original magnification 100x) showing anastomosing cords of basaloid cells with overlying connection to epidermis and stromal retraction. (b) Immunohistochemical staining for smooth muscle actin (original magnification 100x) showing diffuse, strong positivity. (c) Immunohistochemical staining for Ber-EP4 (original magnification 100x) showing diffuse, strong, membranous positivity.

strong and diffuse positivity, further supporting the diagnosis (Figure 1(c)). The patient responded well to excision of the lesion and no further treatment was required.

\section{Discussion}

Basal cell carcinoma of the perianal skin is a rare entity that is most frequently reported by individual case reports or case series. There is a male predominance and the mean age of onset is 67 years [4]. It may vary in its clinical presentation from erythematous papules and patches to nodules, plaques, and ulcers that may present with bleeding, pain, itching, and mucoid discharge $[1,5]$. The majority of these lesions are smaller than $2 \mathrm{~cm}$ and are located outside the anal verge [4]. This location may be useful in distinguishing perianal BCC from basaloid squamous cell carcinoma (BSCL). BSCL is a common anal tumor and most commonly occurs in the anal canal or anorectum (inside the anal verge) [6]. Although several subtypes of BCC exist, the nodular type is the most common in the perianal region [6].

The etiology of perianal BCC remains unknown; however chronic irritation, trauma, immunosuppressive medications, and radiation have been suggested as causative factors [1]. Although most patients with perianal BCC do not have a personal history of tanning bed use or nude sunbathing, approximately $1 / 3$ have a history of skin cancer in sun exposed areas $[1,3]$. This association suggests a predisposition in individuals susceptible to cutaneous malignancies. Therefore, any history of cutaneous malignancy should further prompt clinicians to examine nonsun exposed areas on full body skin exams. Furthermore, frequent BCC development has been associated with an increased overall cancer risk; therefore occurrence of BCC more than once in the same patient should prompt close surveillance for other malignancies (our patient had a history of gastric lymphoma) [7].

It is important to obtain histologic verification of the disease in order to distinguish it from more aggressive malignancies with higher capacity for metastasis such as BSCL. The classic histology of perianal BCC reveals peripheral palisading and peritumoral slits [4]. Immunohistochemical staining is also useful as perianal BCC usually displays diffuse and strong staining for SMA and Ber-EP4 $[6,8,9]$, while BSCL does not [9]. Positive immunohistochemical reactivity with pancytokeratin, EMA, and CEA is not consistent with perianal BCC and may suggest a diagnosis of BSCL [9]. 
According to The National Comprehensive Cancer Network, a BCC in the perianal region is considered 'high-risk' and Mohs Micrographic surgery (MMS) is recommended, although standard $4 \mathrm{~mm}$ margin excision can be considered for select tumors [10]. The treatment of choice for perianal BCC has traditionally been local excision to clear margins, especially for lesions that are less than $2 \mathrm{~cm}$ [4]. Local excision has proven to be the extremely successful since there is usually no recurrence of disease [1,4]. Larger lesions may require skin grafting for closure and only those involving the anal canal or extending into the surrounding tissues require abdominoperineal resection of the rectum $[4,5]$. Our patient exhibited a lesion that was $0.5 \mathrm{~cm}$ in size and excisional biopsy was sufficient treatment for his disease.

Since perianal BCC is so uncommon and is located in an inconspicuous skin area, it may be easily overlooked by clinicians. Therefore, we recommend that gluteal and genital areas be included in full body skin exams, particularly in higher risk individuals. Suspicious lesions should be biopsied and immunohistochemical staining performed to establish the diagnosis.

In summary, perianal BCC is a nonaggressive malignancy with excellent prognosis following local excision to clear margins. Specifically, for perianal lesions smaller than $1 \mathrm{~cm}$, excisional biopsy may be sufficient and better tolerated than MMS or standard local excision with $4 \mathrm{~mm}$ margins. This case report supports the notion that proper clinical vigilance and early detection of perianal BCC can result in excellent prognosis.

\section{Conflicts of Interest}

The authors declare that there are no conflicts of interest regarding the publication of this paper.

\section{Acknowledgments}

We thank the patient for granting permission to publish this information.

\section{References}

[1] G. E. Gibson and I. Ahmed, "Perianal and genital basal cell carcinoma: a clinicopathologic review of 51 cases," Journal of the American Academy of Dermatology, vol. 45, no. 1, pp. 68-71, 2001.

[2] R. Betti, C. Bruscagin, E. Inselvini, and C. Crosti, "Basal cell carcinomas of covered and unusual sites of the body," International Journal of Dermatology, vol. 36, no. 7, pp. 503-505, 1997.

[3] M. L. Welton and M. G. Varma, "Anal cancer," in The ASCRS Textbook of Colon and Rectal Surgery, B. G. Wolff, J. W. Fleshman, D. E. Beck, J. H. Pemberton, and S. D. Wexner, Eds., pp. 482-500, Springer, New York, NY, USA, 1st edition, 2007.

[4] C. A. Paterson, T. M. Young-Fadok, and R. R. Dozois, "Basal cell carcinoma of the perianal region: 20-year experience," Diseases of the Colon \& Rectum, vol. 42, no. 9, pp. 1200-1202, 1999.

[5] O. V. Nielsen and S. L. Jensen, "Basal cell carcinoma of the anus-a clinical study of 34 cases," British Journal of Surgery, vol. 68 , no. 12 , pp. 856-857, 1981.
[6] D. T. Patil, J. R. Goldblum, and S. D. Billings, "Clinicopathological analysis of basal cell carcinoma of the anal region and its distinction from basaloid squamous cell carcinoma," Modern Pathology, vol. 26, no. 10, pp. 1382-1389, 2013.

[7] H. Cho, K. Kuo, S. Li et al., "164 Frequent basal cell cancer development is a clinical marker for inherited cancer susceptibility," Journal of Investigative Dermatology, vol. 138, no. 5, p. S28, 2018.

[8] M. M. Christian, R. L. Moy, R. F. Wagner, and A. Yen-Moore, "A correlation of alpha-smooth muscle actin and invasion in micronodular basal cell carcinoma," Dermatologic Surgery, vol. 27, no. 5, pp. 441-445, 2001.

[9] M. C. Alvarez-Cañas, F. A. Fernández, I. G. Rodilla, and J. F. ValBernal, "Perianal basal cell carcinoma: a comparative histologic, immunohistochemical, and flow cytometric study with basaloid carcinoma of the anus," American Journal of Dermatopathology, vol. 18, no. 4, pp. 371-379, 1996.

[10] J. Y. Kim, J. H. Kozlow, B. Mittal et al., "Guidelines of care for the management of basal cell carcinoma," Journal of the American Academy of Dermatology, vol. 78, no. 3, pp. 540-559, 2018. 


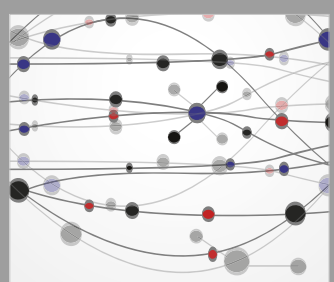

The Scientific World Journal
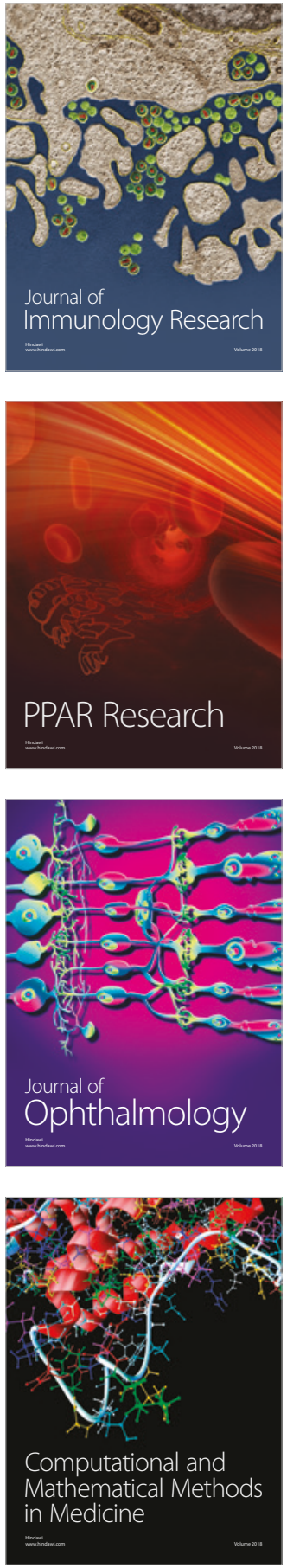

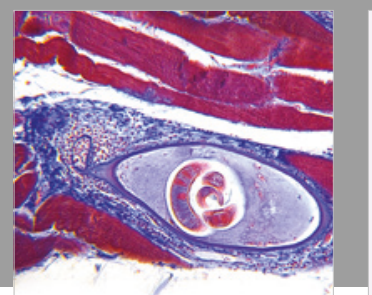

Gastroenterology Research and Practice

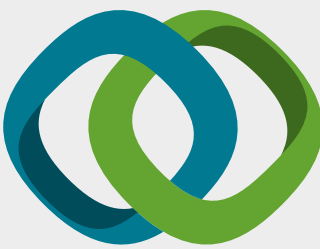

\section{Hindawi}

Submit your manuscripts at

www.hindawi.com
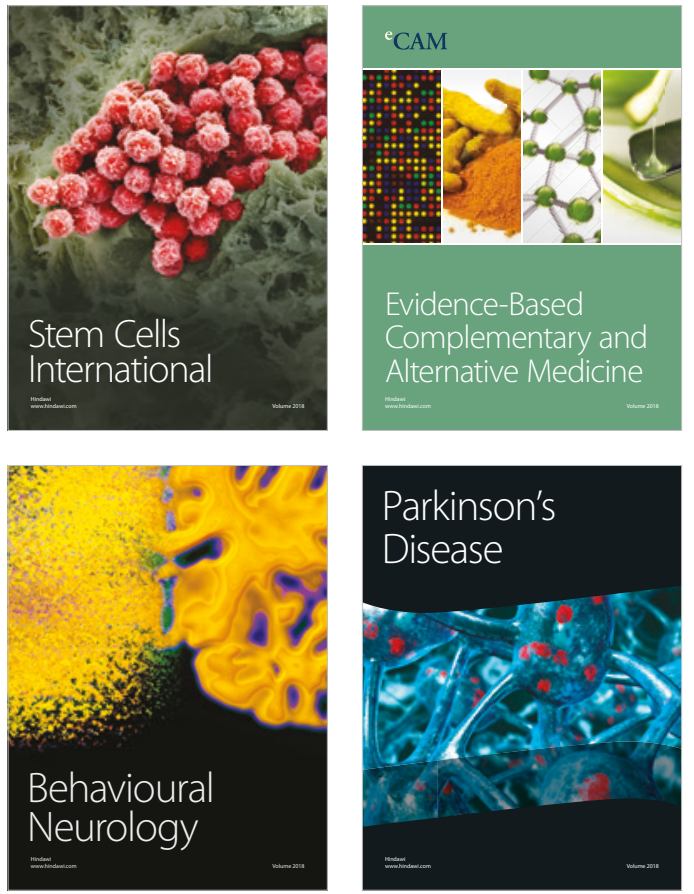

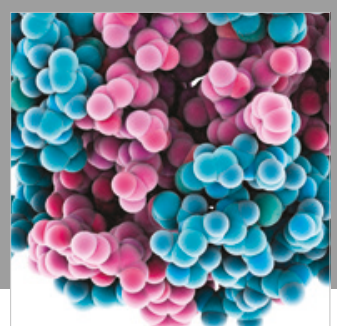

ournal of

Diabetes Research

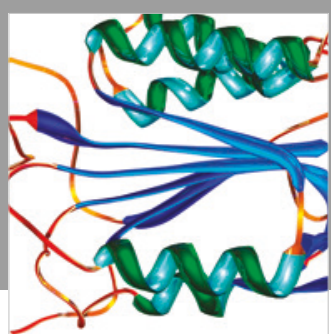

Disease Markers
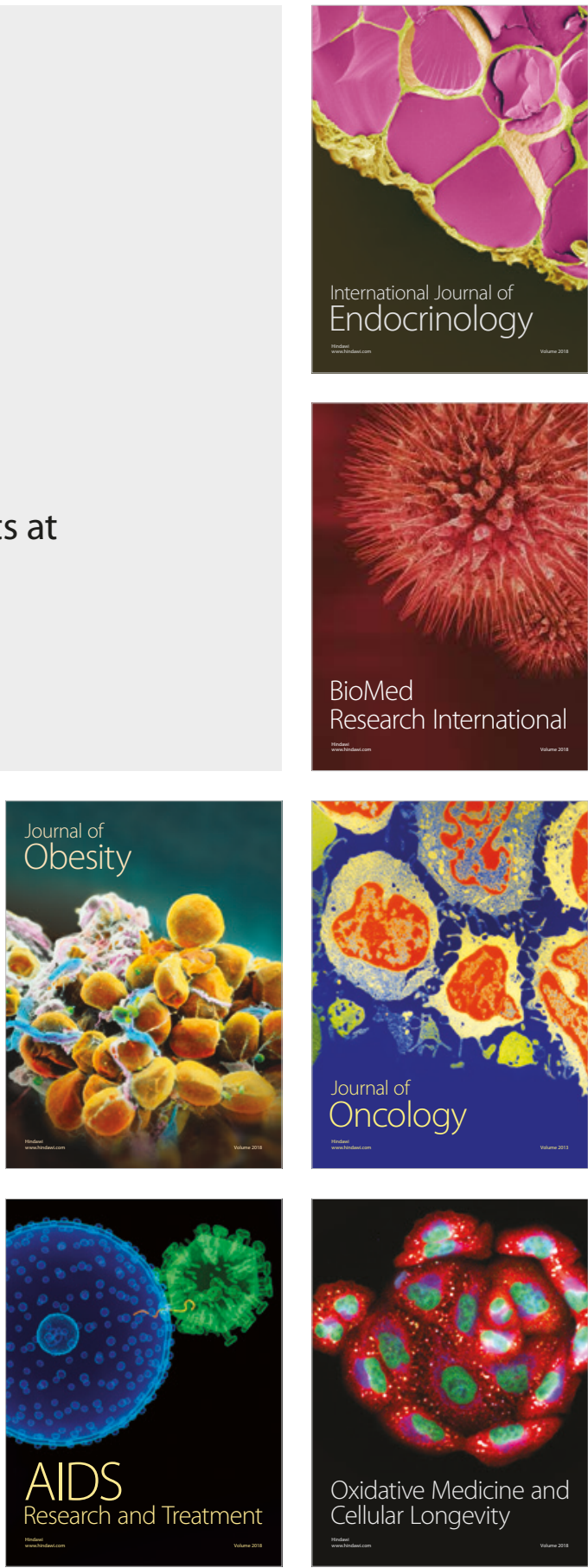